\title{
Quizzen für den Unternehmenserfolg: Weiterbildung mittels Enterprise Social Networks neu gestalten
}

\author{
Julia Friedrich $\cdot$ Christian Zinke-Wehlmann • Julia Holze • \\ Frank Hoffmann
}

Eingegangen: 6. September 2018 / Angenommen: 13. Dezember 2018 / Online publiziert: 17. Januar 2019 (C) Der/die Autor(en) 2019

Zusammenfassung Die Etablierung von Enterprise Social Networks (ESN) ermöglicht es Unternehmen, Kollaborationsarbeit plattformgestützt zu gestalten. Neben wertvollen Funktionen wie Wissensmanagement, Projektmanagement und Unternehmenskommunikation bieten ESN auch Ansatzpunkte zur Neugestaltung der betrieblichen Weiterbildung. Der vorliegende Beitrag untersucht am Beispiel von yeepa ${ }^{\circledR}$ Möglichkeiten innovativer Formen des Lernens durch ESN. Yeepa ${ }^{\circledR}$ ist ein für soziale Netzwerke entwickeltes Wissensquiz, welches individuelle Analysen der Wissensentwicklung und Ansätzen bedarfsgerechter Inhaltsvermittlung bereithält, ohne dabei die Wissensentwicklung der gesamten Spielergemeinschaft zu vernachlässigen.

Im Fokus der nachfolgenden Betrachtung steht vor allem die Notwendigkeit der Analyse und Gestaltung von Rahmenbedingungen des Spielens. Das Ziel von Gamification im Kontext der betrieblichen Weiterbildung liegt darin, eine zum Lernen nachhaltig motivierende und im Hinblick auf Mitarbeiterqualifizierung effiziente Anwendung zu schaffen. Je attraktiver und motivierender eine solche Anwendung ist, desto größer ist zugleich die Gefahr, dass sich der Fokus der Mitarbeiter weg von der primären Arbeitstätigkeit hin zum spielbasierten Lernen verschiebt. Definierte Prozesse und Strukturen sind deshalb vonnöten, um die motivierende ESN-basierte

\footnotetext{
J. Friedrich $(\bowtie) \cdot$ C. Zinke-Wehlmann $\cdot$ J. Holze Institute for Applied Informatics (InfAI) e. V., Goerdelerring 9, 04109 Leipzig, Deutschland E-Mail: friedrich@infai.org

C. Zinke-Wehlmann

E-Mail: zinke@infai.org

J. Holze

E-Mail: holze@infai.org

F. Hoffmann

SNTL Publishing GmbH \& Co KG, Hegelplatz 1, 10117 Berlin, Deutschland

E-Mail: fh@sntl-publishing.com
} 
Wissensvermittlung ohne die Gefahr einer Fokusverschiebung eindeutig im Arbeitsalltag zu verankern. Dazu müssen beispielsweise Fragen nach der zeitlichen Dimension für spielbezogene Aktivitäten ebenso wie nach Varianten der Anreizvergabe für Lernerfolge Berücksichtigung finden. Hierzu werden nachfolgend verschiedene Spielsettings konzipiert und im Hinblick auf Chancen und Risiken analysiert.

Schlüsselwörter Gamification · Enterprise Social Networks · kollaboratives Lernen · betriebliche Weiterbildung · Design Research

\title{
Quiz Playing for Corporate Success: Redesigning Corporate Training Using Enterprise Social Networks
}

\begin{abstract}
The adoption of Enterprise Social Networks (ESN) enables companies to design platform supported collaboration processes. In addition to valuable functions such as knowledge management, project management, and communication, ESN also offer starting points for redesigning corporate training. This article examines the possibilities of innovative forms of learning through ESN using the example of yeepa ${ }^{\circledR}$, which is a knowledge quiz developed for social networks. Yeepa ${ }^{\circledR}$ provides individual knowledge development analyses and approaches to need-based training by a targeted content provision, without neglecting the knowledge development of the entire player community. Above all, the focus of the following consideration is the necessity of analyzing and designing the framework conditions of game-based learning. The goal of gamification in the context of corporate training is to create an application that motivates learning in the long term and is efficient with regard to employee qualification. The more attractive and motivating such an application is, the greater is the risk that the focus of the employees' shifts away from the primary work activity to game-based learning. To this end, it is necessary, for example, to take the time dimension for game-related activities into consideration as well as variants of the allocation of incentives to support learning success. To this end, various game settings will be designed and analyzed with regard to opportunities and risks.
\end{abstract}

Keywords Gamification · Enterprise social Networks · Collaborative learning · Corporate training $\cdot$ Design research

\section{Ansätze spielbasierter Weiterbildung im Unternehmenskontext}

Eine kontinuierliche Kompetenzentwicklung gilt als Grundlage für die Innovationsfähigkeit eines Unternehmens. Neben Wissensmanagement-Prozessen, die als Voraussetzung gelten, um betriebsrelevantes Wissen systematisch zu erhalten, sind Weiterbildungsangebote notwendig, um die Wissensaneignung von Mitarbeitern zu unterstützen. Neben technischen und organisatorischen Rahmenbedingungen ist die Motivation der Mitarbeiter, als Quelle und Rezipient von Wissen, eine entscheidende Herausforderung im Hinblick auf einen nachhaltigen Umgang mit Wissen in Unternehmen (Ardichvili et al. 2003). 
Neue digitale Medien, intelligente Vernetzung von Informationen und die damit einhergehenden Möglichkeiten der Wissensgenerierung und -vermittlung ermöglichen es, traditionelle Vermittlungskonzepte aufzubrechen und Lernen selbstgesteuert und am individuellen Bedarf orientiert zu gestalten. Dabei rücken immer häufiger auch Enterprise Social Networks (ESN) in den Fokus. ESN sind webbasierte Plattformen, welche die Kommunikation, Kollaboration und Wissensvermittlung zwischen Angestellten eines Unternehmens ermöglichen (McAfee 2006). Die soziale Interaktion über ESN ist zeit- und ortsunabhängig, sodass Mitarbeiter ${ }^{1}$ auch über Ländergrenzen und Zeitzonen hinweg miteinander in Verbindung bleiben können. Unternehmen implementieren ESN häufig mit dem Ziel die Produktivität zu erhöhen oder Innovationen voranzutreiben. Doch wenngleich das Potential von ESN als Plattform für den innerbetrieblichen ebenso wie überbetrieblichen Wissensaustausch zwischen Akteuren erkannt wurde, beschränken sich die konkreten Anwendungsszenarien häufig auf ESN-Standardfunktionalitäten (Sharing, Tagging, Micro-Blogging, Search, etc.). Dabei stehen informelle Lernprozesse im Vordergrund der Betrachtung (Scott et al. 2016). Ansätze einer gezielten Steuerung von Weiterbildungsprozessen durch eine Verknüpfung von ESN mit spezifischen Weiterbildungstools für den betrieblichen Kontext gibt es dagegen kaum. Im vorliegenden Beitrag soll mit yeepa ${ }^{\circledR}$ ein zum Zwecke von Training und Wissensentwicklung konzipiertes Social Serious Game vorgestellt und Chancen wie auch Möglichkeiten einer Einbindung in ESN diskutiert werden. Zur Stärkung des terminologischen Verständnisses folgt jedoch zunächst eine Abgrenzung zwischen Gamification und (Social) Serious Game.

\subsection{Gamification und Serious Games in der Wissensvermittlung}

Gamification als die Einbindung spielerischer Elemente in einen spiel-fremden Kontext (Deterding et al. 2011) bietet vielfältige Möglichkeiten, gezielte Anreize zur Ausführung von Aktivitäten zu setzen. So können etwa Punktesysteme, Badges oder andere Belohnungsmechanismen unter entsprechendem Implementierungsaufwand in (ggf. bereits existierende) Systeme eingebunden werden, um spielerische Effekte von Herausforderungen, Wettkämpfen oder Status zu nutzen. Der Erfolg von Gamification hängt zudem entscheidend vom Design und der Implementierung in den entsprechenden Lern- oder Arbeitskontext (Rahmenbedingungen) ab.

Bei Serious Games handelt es sich um konkret mit dem Ziel der Wissensvermittlung (z.B. Vokabeltrainer) oder zum Trainieren spezieller Fähigkeiten (z.B. Flugsimulator) entwickelte Spiele. Um den Effekt von Spielen auf die Motivation der Nutzer zu verstehen, wird oft auf die von Csikszentmihalyi (1990) entwickelte Flow Theorie zurückgegriffen, welche den Zusammenhang von Herausforderung und Fähigkeiten eines Spielers sowie deren Einfluss auf das Spielerlebnis darstellt. Ziel eines Spiels muss es sein, sowohl Überforderung als auch Langeweile zu vermeiden, um den Spieler engagiert und „bei Laune“ zu halten. Ein Flow-Erlebnis stellt sich ein, wenn die Balance zwischen dem Schwierigkeitsgrad einer Aufgabe und den Fähigkeiten eines Spielers ausgeglichen ist. Charakteristisch für ein FlowErlebnis sind demnach das Vertiefen in die Arbeitsaufgabe, einhergehend mit hoher

\footnotetext{
1 Aus Gründen der Lesbarkeit wird im Text verallgemeinernd das generische Maskulinum verwendet.
} 
Konzentration und Fokussierung sowie einer gesteigerten Empfänglichkeit für Informationen, einem Verlust der Wahrnehmung von Zeit und Umgebung, intrinsische Motivation und hohes Engagement. Zu den Voraussetzungen für ein solches Empfinden zählen eine klare Aufgabe mit entsprechenden Zielen, sofortiges Feedback, Selbstbestimmtheit und ein starkes, aber unangestrengtes Engagement. Bezogen auf Serious Games bedeutet das, dass die Fähigkeiten und Kompetenzen eines Spielers stets berücksichtigt werden müssen, um die Motivation für das Spielen bzw. Lernen hoch zu halten, und dass demnach eine gewisse inhaltliche Dynamik im Spiel vorhanden sein muss. Ein Spielsetting beinhaltet alle relevanten Aspekte, welche direkten oder indirekten Einfluss auf das Spiel und die Motivation haben.

Werden die Spiele in den Kontext kollaborativer Software (Groupware) eingebettet, sprechen wir von Social Serious Games. Weiterbildungskonzepte auf Basis von Social Serious Games erfordern die aktive Beteiligung der Mitarbeiter, welche die ihnen im ESN-Kontext zur Verfügung gestellten Weiterbildungsangebote autonom und selbstbestimmt nutzen sollen. Um ihnen dies $\mathrm{zu}$ ermöglichen, sind zunächst jedoch die passenden Rahmenbedingungen zu schaffen.

\section{Methode}

Im vorliegenden Fall wird das Ziel verfolgt, die betriebliche Weiterbildung mit alltäglichen Arbeitsprozessen zu verknüpfen. Spielerische Elemente und die Nutzung gruppendynamischer Effekte sollen dazu beitragen, die generelle Motivation zur Weiterbildung zu erhöhen. Dazu bedarf es Vorgehensmodellen und konkreten Lösungsansätzen. Die im vorliegenden Fall gewählte Methode basiert auf vier der von Peffers et al. (2007) beschriebenen Schritte des Design Science Research Ansatzes. Diese sind:

- I: Problem beschreiben

- II: Lösungsansatz entwickeln

- III: Design und Implementierung Artefakt

- V: Evaluation (und ggf. Einleiten eines weiteren Design-Zyklus')2

Der beschriebene Prozess ist fortlaufend und mehrstufig. Mit yeepa ${ }^{\circledR}$ liegt ein Artefakt mit ersten Implementierungserfahrungen vor, welches dem Problem der mangelnden Lernmotivation durch eine quizbasierte Aufbereitung von Lerninhalten und die individuelle Analyse und Darstellung des Lernfortschritts begegnet. Bislang wird yeepa ${ }^{\circledR}$ im Rahmen zeitlich begrenzter Projekte als isolierte Spiellösung (Serious Game) eingesetzt. In einer weiteren Stufe des Designprozesses soll dieser Projektcharakter nun aufgebrochen und eine sowohl kontinuierliche als auch nachhaltige Qualifikation von Mitarbeitern im alltäglichen Arbeitsprozess etabliert werden. Dabei ist yeepa ${ }^{\circledR}$ nach wie vor ein in sich geschlossenes Spiel, welches durch die Einbindung in den ESN-Kontext jedoch einen stärkeren Netzwerkcharakter erhält (Social Serious Game) und dadurch den kollaborativen Aspekt der betrieblichen

\footnotetext{
2 Peffers behandelt ursprünglich 6 Schritte. Im vorliegenden Fall wird der Schritt Demonstration (IV) unter Evaluation subsummiert. Schritt VI ist die Kommunikation der Erkenntnisse aus dem Gesamtprozess.
} 
Weiterbildung stärkt. Die theoretische Grundlage bilden wissenschaftliche Erkenntnisse aus dem Bereich der Mensch-Technik-Organisations-Analyse. Die zentrale Herausforderung liegt in der Analyse und Gestaltung der Rahmenbedingungen und der Entwicklung konkreter Spielsettings. Kurz: Wie ist es möglich ein Social Serious Game erfolgreich in Unternehmen zu integrieren? Im Folgenden werden zunächst Spielaufbau und Weiterbildungskonzept von yeepa ${ }^{\circledR}$ erläutert. Nachfolgend wird ein Lösungsansatz beschrieben, welcher die Rahmenbedingungen klassifiziert und konkrete Implementierungsansätze in Form von Spielsettings beschreibt. Die Evaluation (inkl. Demonstration) findet im Rahmen des Förderprojektes SB:Digital statt und steht noch aus.

\section{3 yeepa $^{\circledR}$ : Spielen - Messen - Lernen}

yeepa ${ }^{\circledR}$ ist eine skalierbare, client-server-basierte Web-App zur Produktion, Organisation und Durchführung von Echtzeit-Multiplayer-Quiz-Szenarien. Ein breit akzeptiertes Spielformat, wie das des Quiz', kann durch die Vernetzung der Spieler und die dadurch evozierte Intensivierung der Nutzung sowie die spielbedingte Wiederholung der Quizfragen quasi en-passant sehr große Mengen an Verlaufsdaten erzeugen.

Das Spielprinzip ist einfach: Nach der Registrierung kann der Spieler in für freigeschaltete Quiz-Games einsteigen. Dabei spielt er alleine oder gegen andere Spieler um Punkte. Je mehr Spieler sich im Spiel befinden, umso mehr Punkte lassen sich gewinnen, denn die Gesamtpunktzahl errechnet sich aus Antwortpunkten (entsprechend Antwortverhalten und Fragenschwierigkeit) und Jackpot-Punkten. Der erzielte Wert der Antwortpunkte verhält sich umgekehrt proportional zur benötigten Antwortzeit. Die Punkte aller Spieler, die falsch geantwortet haben, wandern in den Jackpot. Der Wechsel zur nächsten Frage passiert bei allen Mitspielern gleichzeitig. Per Zufall entscheidet das System darüber, ob eine Frage zur Jackpot-Frage wird. Durch die für Spiele charakteristischen Einflussfaktoren Zufall und Strategie wird die Variabilität des Spiels erhöht und eine Dramaturgie erzeugt. Zugleich stellt das Jackpot-Element auch eine Möglichkeit dar, Spielern mit (anfangs) niedrigerem Wissensstand Erfolgserlebnisse in Form hoher Score-Werte zu ermöglichen und dadurch Demotivation vorzubeugen. Nach üblicherweise sechs Fragen endet das Spiel mit der Visualisierung des Spielerfolgs, inkl. Hinweisen zur Spiel-optimierung (LernTipps). Diese Lerntipps werden individuell auf Basis des Antwortverhaltens erstellt, wodurch Lerninhalte bedarfsgerecht zur Verfügung gestellt werden.

Die Leistungs-Messung geschieht dabei auf zwei unterschiedlichen Ebenen:

- Gaming-Layer: Der Spielerfolg wird mittels Spielpunkten gemessen und in Form von High-Scores ausgewertet. Der Score eines Spielers ergibt sich aus den Antwort- und Jackpot-Punkten.

- Serious-Layer: Die Wissensentwicklung wird anhand von Wissensindexen gemessen und dem Spieler visualisiert. Grundlage dafür bildet die Analyse des Antwortverhaltens bei der binär zwischen Wissen und Nicht-Wissen unterschieden wird. Für Organisatoren ist die Wissensentwicklung aggregiert auf Gruppenebene sichtbar. 
Im Gegensatz zu herkömmlichen Werkzeugen zur Berechnung von Wissenskapital, welche lediglich ungefähre Interpretationen erlauben, ist yeepa ${ }^{\circledR}$ in der Lage, kontinuierlich eintreffende Spieldaten auf der mathematischen Grundlage der Item Response Theory (IRT) zu analysieren und Spielleistungen zu einem instruktiven Indexwert zu kondensieren. Dieser yeepa-Index repräsentiert quasi die Entwicklung des Wissenskapitals.

Begreift man Lernen als sozialen Prozess (Michl 2015), dann setzt yeepa ${ }^{\circledR}$ mit der psychometrischen Validierung von Kommunikations- und Lernzielen an folgenden Punkten an:

- Intrinsische Motivation: spielerischer Wettbewerb mit anderen

- Extrinsische Motivation: soziale Peer-Effekte (Interaktion), Preise

- Selbsterfahrung: Wissensstandmessung

- Türöffner-Setting: Anregung sachorientierter (Offline-)Diskussionen unter Nutzern

- Selbststeuerung: selbstbestimmtes Spielen

Bei der konkreten Einführung von yeepa ${ }^{\circledR}$ in eine Organisation oder ein Projekt hat sich bislang gezeigt, dass eine Mehrheit der Spieler auf extrinsische Motivation angewiesen ist. D.h. die Motivation steigt, wenn die Spielergebnisse entweder als Voraussetzung für eine andere Maßnahme herangezogen werden oder es kleine, reale Preise, idealerweise verbunden mit einer Preisvergabe mit sozialem Rahmen, zu gewinnen gibt.

Im Folgenden werden hieran anknüpfende Überlegungen hinsichtlich einer netzwerkbasierten, sozialen Motivation zur Nutzung der Wissensanwendung yeepa ${ }^{\circledR}$ dar- $^{-}$ gestellt.

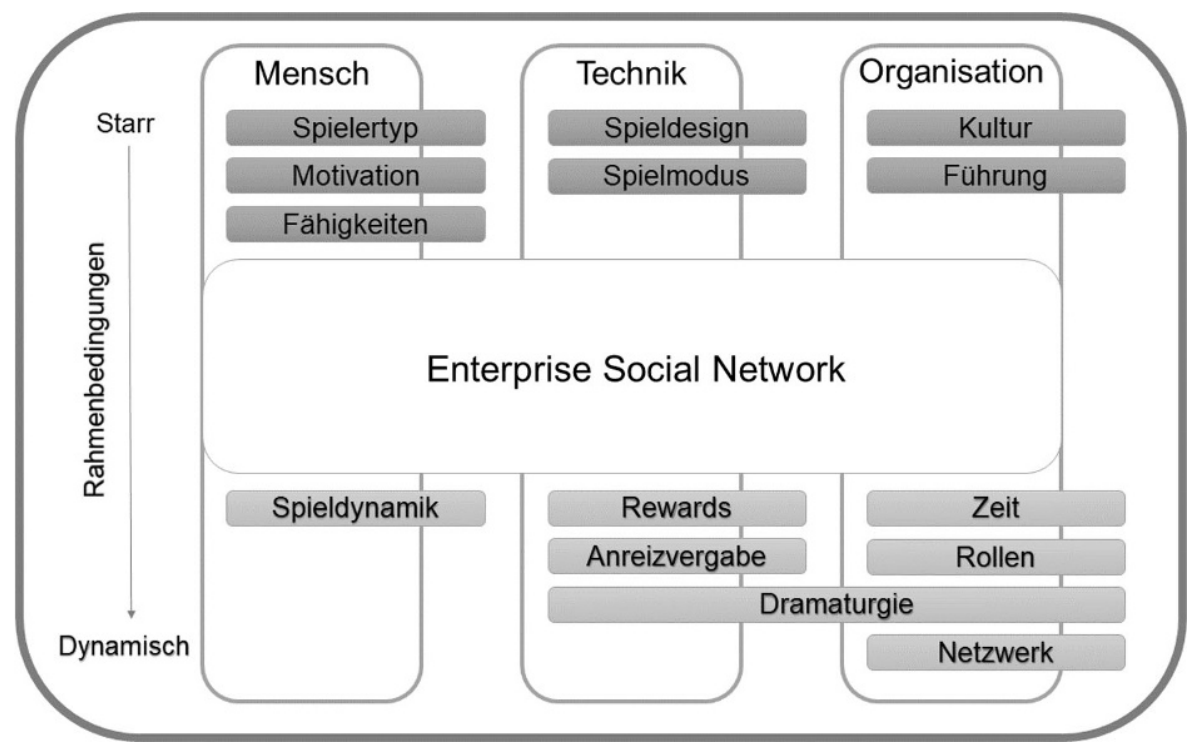

Abb. 1 Rahmenbedingungen für Social Serious Games 


\section{Rahmenbedingungen für die Einbindung von Social Serious Games in Unternehmen}

Das Ziel einer Einbindung von yeepa ${ }^{\circledR}$ in die Unternehmensprozesse ist die Etablierung nachhaltiger und an den individuellen Bedarf der Mitarbeiter angepasster Weiterbildung, welche permanent verfügbar und dadurch in den beruflichen Alltag integrierbar ist. Das Prinzip des Spiels basiert auf Lernen durch Wiederholung. Die Herausforderung besteht deshalb darin, die Spieler in einen Flow-Zustand zu versetzen und sie durch den Einsatz von Spielmechanismen und -dynamik dazu zu bewegen, möglichst kontinuierlich zu spielen und dies zugleich mit den begrenzten zeitlichen Ressourcen für Weiterbildungsmaßnahmen in Einklang zu bringen. Gegebenenfalls müssen Intensivspieler also sogar gebremst werden, ohne dass für sie der Reiz des Spiels verloren geht. Das heißt, es sind klare Rahmenbedingungen für die Einbindung von Social Serious Games in den Arbeitsalltag erforderlich, um sowohl den Anforderungen der Geschäftsführung als auch denen der Mitarbeiter gerecht zu werden.

In der Gestaltung von Rahmenbedingungen bedienen wir uns des, in der Betrachtung soziotechnischer Systeme gängigen, MTO-Konzepts (Ulich 2013). Dabei wird grundsätzlich zwischen dynamischen und starren Aspekten unterschieden. Die in Abb. 1 überblicksartig dargestellten Rahmenbedingungen werden im nachfolgenden Abschnitt erläutert, wobei aufgrund des erhöhten Gestaltungsspielraumes ein klarer Schwerpunkt auf die Beschreibung der dynamischen Aspekte gelegt wird.

\subsection{Der Spieler (menschbezogener Rahmen)}

Der Erfolg eines Serious Game ist auf Ebene des Menschen abhängig von der intrinsischen Motivation eines Spielers, welche maßgeblichen Einfluss auf dessen Spielbereitschaft hat. Ansätze zur Typisierung von Spielertypen ${ }^{3}$ verdeutlichen, dass sich Menschen ganz individuell durch unterschiedliche Spielmechanismen besonders motivieren lassen. Während Spielertyp ebenso wie individuelle Fähigkeiten starre Aspekte sind, lässt sich die Spieldynamik, also das sich aus der Spielgestaltung individuell ergebende Spielerlebnis und -engagement, durch den gezielten Einsatz von Anreizen zumindest indirekt steuern (Shpakova et al. 2016).

\subsection{Das Spiel (technischer Rahmen)}

Auf technischer Ebene ist neben dem grafischen Spieldesign der Spielmodus (Singleoder Multiplayer) als relativ starre Rahmenbedingung zu nennen, welche meist nur mit hohem Aufwand geändert werden kann. Dynamisch sind hingegen die zu wählenden Rewards sowie die Anreizvergabe.

\footnotetext{
3 Bartle (1996) unterscheidet die vier Spielertypen Achiever, Socialiser, Explorer, Killer. Das BrainHex Modell unterscheidet die sieben Spielertypen: Seeker, Survivor, Daredevil, Mastermind, Conqueror, Socialiser und Achiever (Nacke et al. 2011).
} 
Jegliche Formen der Belohnung bzw. öffentlichen Auszeichnung von Leistungen werden im Kontext von Gamification als Rewards bezeichnet. Diese können materieller sowie immaterieller Natur sein. So sind etwa virtuelle Badges eine Form der Anerkennung. Badges vermitteln dem Empfänger ein Gefühl von Kompetenz und dienen auch als Vergleichskriterien zwischen Spielern. Auch Ranglisten dienen dem Vergleich und sollen Motivation durch Ehrgeiz fördern. Um negative Effekte, wie Demotivation durch Darstellung weniger guter Leistungen, zu vermeiden, sollten jedoch anonymisierte Ranglisten oder Bestenlisten angezeigt werden.

Rewards oder Belohnungssysteme dienen dazu, Verbundenheit mit einem System zu erzeugen und es für den Nutzer möglichst abwechslungsreich und interessant zu gestalten. Das spielerische Element des Einsammelns von Badges oder erreichen von Levels spricht insbesondere die Spielertypen Achiever (möchte möglichst viel der möglichen Errungenschaften erreichen) und Explorer (möchte die virtuelle Spielumgebung möglichst umfassend erkunden, alles ,,erleben“) an. Rewards, die auf der Grundlage von Feedback aus der Nutzergruppe heraus vergeben werden (z. B. Badges für eine bestimmte Anzahl von positiv bewerteten Quizfragen, die von einem Spieler erstellt wurden) sowie andere Formen der Interaktion zwischen Spielern (z.B. Spieleinladungen oder Likes für Errungenschaften anderer) sind besonders interessant für den Spielertyp des Socializers, welcher bevorzugt mit Mitspielern in Kontakt tritt. Ehrgeizige Spielertypen wie Killer oder Conquerer profitieren von Netzwerkaktivitäten, indem sie beispielsweise die Möglichkeit erhalten, Konkurrenten direkt zum Spiel herauszufordern und ggf. zu besiegen.

Im Unternehmenskontext ist es auch vorstellbar, Achievements wie Badges oder Ranglistenplatzierungen in materielle Anreize umzuwandeln. Diese können sowohl monetär (Geldleistung) als auch nicht-monetär (Freizeitausgleich) sein. Da materielle Anreize auf lange Sicht jedoch die Gefahr einer Verdrängung intrinsischer Motivation bergen, sollte ihr Einsatz stets genau geprüft werden.

Um die Wirksamkeit rein virtueller Anreize wie Badges und Ranglisten zu bewahren, ist es notwendig, zu variieren und dynamische Formen der Anreizvergabe zu wählen. Chou (2013) listet die folgenden sechs Vergabearten von Rewards auf:

- Fixed action reward (klares Ziel): Die Konditionen für den Erhalt des Rewards sind klar definiert und allen Teilnehmern bekannt.

- Beispiel: Buy 1, get 1 free

- Random reward (Zufall): Potentielle Empfänger wissen, dass es einen Reward gibt, kennen den Inhalt aber nicht. Es geht um Spannung und die Freude am überrascht werden.

- Beispiel: Loskauf (Niete oder Gewinn)

- Sudden reward (Überraschung): Es gibt einen unbekannten Trigger für den Erhalt eines Reward.

- Beispiel: Der 1000 Kunde erhält einen Blumenstrauß

- Rolling reward (Chance): Es gibt eine Gewinnchance durch Erreichen eines definierten Zieles.

- Beispiel: Newsletter anmelden, für Gewinnchance auf Prämie

- Social Treasure (Dank): Gegenseitig von Spielern vergebene Belohnungen.

- Beispiel: Likes oder positive Bewertungen, Votings 
- Price Packing (Sammeln): Einsammeln von Dingen/Erfüllen festgelegter Aufgaben bzw. Bedingungen in einem bestimmten Zeitraum, um eine zuvor bereits bekannte Belohnung zu erhalten

- Beispiel: Bonuskarte: 12 Kaffee kaufen, den 13. gibt es kostenlos

In Tab. 1 werden konkrete Rewards skizziert, welche die Verknüpfung von Spielund Arbeitsprozess unterstützen und den Charakter von yeepa ${ }^{\circledR}$ als Social Serious Game stärken können.

Erzielte Errungenschaften wie Badges können von den Spielern gesammelt werden, in ihrem privaten Spielerprofil verborgen oder im ESN geteilt werden. Das öffentliche Präsentieren von Badges erlaubt es Spielern, Anerkennung für ihre Spielleistung zu erhalten und bedient dadurch Statusbestrebungen. Eine weniger konkurrenzbetonende Form der Motivation, lässt sich durch die Einbindung von Badge Collections erreichen. Bei einer Badge Collection sind alle erreichbaren fixed action rewards für den Mitarbeiter sichtbar, müsssen jedoch durch Erreichen der entsprechenden Kennzahlen freigespielt werden. Hierdurch wird der Sammeleifer von Spielern zur Motivation fürs Weiterspielen.

Auch Dashboards können Anreize für Spieler setzen, indem sie Feedback auf verschiedenen Ebenen geben, Eine Übersicht über Möglichkeiten zur Integration von Dashboards in yeepa ${ }^{\circledR}$ liefert Tab. 2.

Tab. 1 Möglichkeiten für Rewards in yeepa ${ }^{\circledR}$

\begin{tabular}{|c|c|c|c|}
\hline Art & Bezeichnung & $\begin{array}{l}\text { Reward } \\
\text { Vergabe }\end{array}$ & Kennzahl \\
\hline Spielpunkte & Score & $\begin{array}{l}\text { fixed } \\
\text { action }\end{array}$ & $\begin{array}{l}\text { Übersicht der erzielten Spielpunkte als sofortiges } \\
\text { Feedback nach Beendigung der Quizrunde }\end{array}$ \\
\hline \multirow[t]{4}{*}{$\begin{array}{l}\text { Aktivitäts- } \\
\text { punkte }\end{array}$} & gespielte Runde & $\begin{array}{l}\text { fixed } \\
\text { action }\end{array}$ & $\begin{array}{l}\text { je gespielte Runde erhält der Nutzer } 1 \text { Aktivitäts- } \\
\text { punkt }\end{array}$ \\
\hline & gestellte Fragen & $\begin{array}{l}\text { fixed } \\
\text { action }\end{array}$ & $\begin{array}{l}5 \text { Aktivitätspunkte für jede gestellte und ins Spiel } \\
\text { übernommene Quizfrage }\end{array}$ \\
\hline & $\begin{array}{l}\text { regelmäßiges } \\
\text { Spielen }\end{array}$ & $\begin{array}{l}\text { fixed } \\
\text { action }\end{array}$ & $\begin{array}{l}\text { Punkte für regelmäßiges Spielen (mind. } 3 \text { Tagen/ } \\
\text { Woche für } 3 \text { Wochen) }\end{array}$ \\
\hline & Treuebonus & random & $\begin{array}{l}\text { virtuelles „Geschenk“ (Zusatzpunkte) nach } 20 \\
\text { gespielten Runden } \\
\text { per Zufallsprinzip bestimmte Bonushöhe (variie- } \\
\text { rend zwischen } 5 \text { und 20P.) }\end{array}$ \\
\hline \multirow[t]{5}{*}{ Badge } & Treue Seele & $\begin{array}{l}\text { fixed } \\
\text { action }\end{array}$ & $\begin{array}{l}2 \times \text { Einloggen pro Woche an } 4 \text { aufeinanderfolgen- } \\
\text { den Wochen }\end{array}$ \\
\hline & Lucky Looser & sudden & $\begin{array}{l}\text { Yeepa-Index im Top-Drittel, High-Score im Bot- } \\
\text { tom-Drittel }\end{array}$ \\
\hline & Lucky Winner & sudden & $\begin{array}{l}\text { in der Summe mehr Jackpot-Punkte als Eigen- } \\
\text { Punkte kassiert }\end{array}$ \\
\hline & Networker & $\begin{array}{l}\text { fixed } \\
\text { action }\end{array}$ & 10 Spieleinladungen in zwei Wochen versandt \\
\hline & Champion & sudden & $\begin{array}{l}\text { Spieler mit der höchsten in der laufenden Woche } \\
\text { erreichten Punktzahl (verschwindet, sobald ein } \\
\text { anderer Spieler eine höhere Punktzahl erreicht hat) }\end{array}$ \\
\hline
\end{tabular}


Tab. 2 Dashboard-Varianten für yeepa ${ }^{\circledR}$

\begin{tabular}{ll}
\hline Dashboard Typ & Inhalt und Nutzen \\
\hline Game Performance & $\begin{array}{l}\text { unmittelbares Feedback zur Spielleistung nach Beendigung einer Spielrunde } \\
\text { adressiert den Ehrgeiz der Spiele und das Streben nach Verbesserung der eige- } \\
\text { nen Leistung } \\
\text { Messung und Visualisierung der Wissensentwicklung und des Lernfortschritts } \\
\text { Lernfortschritt }\end{array}$ \\
unterstützen den Wunsch des Spielers, die eigene Kompetenz zu steigern \\
Darstellung von Interaktionen innerhalb des Spielernetzwerks sowie Vergleiche \\
mit der Spielergemeinschaft, wie etwa Bestenlisten, spielbezogene Netzwerk- \\
analysen (versandte und angenommene Spieleinladungen) oder ein Vergleich \\
der Spielaktivität, ermöglichen es den Spieler, sich miteinander zu vergleichen
\end{tabular}

Die Dramaturgie ist prägend für das Spielerlebnis. Die technische Umsetzung dramaturgischer Elemente erfolgt über Spielmechanismen, wie Levels, Status und Unlocking (Freischalten von (Bonus-)Inhalten). Durch deren Einbindung in eine Rahmenhandlung (Storytelling) wird ein Spannungsaufbau erzeugt, wodurch reale (Lern-)Ziele spielerisch verpackt werden können. Die Dramaturgie hat somit auch Einfluss auf die individuelle Spieldynamik (Flow-Zustand) und dadurch das Engagement des Spielers.

Konkret auf yeepa ${ }^{\circledR}$ anwendbare dramaturgische Elemente sind:

- Level: Definierte Frageeinheiten zu Themengebieten oder Kompetenzen. Das erfolgreiche Absolvieren eines Levels ist Voraussetzung für das Erreichen eines neuen Levels.

- Berechtigungen: In Abhängigkeit von (Spiel-)Erfahrung bzw. Kompetenz eines Mitarbeiters können Spieler Fragen beantworten, Fragen bewerten oder selbst neue Fragen in den bestehenden Fragenpool einbringen.

- Status: Der Status eines Spielers spiegelt seinen Spielfortschritt (Aktivitätspunkte, Gesamtscore) wieder und ermutigt dazu, die eigene Leistung stetig zu übertreffen, um einen höheren Status zu erreichen. Status können zudem an Berechtigungen geknüpft sein und dadurch zusätzlich den Ehrgeiz der Spieler befeuern.

\subsection{Die Spielumgebung (organisatorischer Rahmen)}

Auf Ebene der Organisation zählen die Unternehmenskultur und -führung zu den starren Aspekten, die Einfluss auf den Erfolg einer Integration von Serious Games in ESN haben. Einführung und Etablierung von Gamification bzw. Ansätzen spielbasierten Lernens sollten strategisch gesteuert und von einer, den offenen Austausch fördernden, Unternehmenskultur umrahmt werden. Zugleich müssen alle Entscheidungsebenen von der Unternehmensführung bis zum Betriebsrat in die Umgestaltung der Arbeits- und Lernprozesse eingebunden werden, um Akzeptanz zu sichern (Stieglitz 2015). Zeitliche Aspekte des Spiels, ebenso wie Rollen, Dramaturgie und Netzwerk sind als dynamische Rahmenbedingungen zu verstehen.

Die Zeit ist für jedes Unternehmen ein kritischer Faktor. Umso wichtiger ist es, sich vor der Einführung eines Social Serious Games über die zeitliche Einbettung des Spiels klar zu werden. Richtig eingesetzt ist ein Social Serious Game wie yeepa ${ }^{\circledR}$ ein 
Mechanismus des aktiven Wissensaustausches, der Wissensgenerierung (und Wiederauffrischung) und Quantifizierung des Wissensstandes im Unternehmen. Ebenso ist es ein Motivator und eine teambildende Maßnahme. All diesen Aspekten muss in der Kalkulation (und Kommunikation) zeitlicher Rahmenbedingungen Rechnung getragen werden. Dabei sind auch unternehmenskulturelle Faktoren von Bedeutung. In einem von selbstbestimmter Arbeitsweise geprägten Umfeld kann es etwa sinnvoll sein, keine starren zeitlich Vorgaben zu machen, sondern lediglich Wissensziele zu formulieren. In restriktiveren Strukturen mit Lehrer-Lernenden-Settings können stattdessen kurze Spielzyklen durch Kampagnen, etwa in Form zeitlich begrenzter Challenges und festen Zeitvorgaben, gestaltet werden. Für beide Settings ist eine Planung und Messung des Zeitaufwandes sinnvoll.

Die Wahl der Settings ist auch mit dem Gestaltungsparameter der Rollen verknüpft. Hierbei steht die Frage im Raum, wie die Rollenstruktur im Unternehmen aussieht und wie diese für die Dramaturgie genutzt werden kann. Durch eine Manifestation oder einen aktiven Verzicht auf Rollen und dem damit einhergehenden Status und Berechtigungen, haben Rollen Einfluss auf die Dramaturgie des Spiels. In einem von flachen Hierarchien geprägten Umfeld spielen etwa alle gemeinsam für den (Lern-)Erfolg der Gemeinschaft.

Der dynamisch gestaltbare Aspekt der Dramaturgie hat Einfluss auf das Spielerlebnis und damit das Engagement der Nutzer. Um dieses hoch zu halten, ist es unbedingt erforderlich, die Spieler immer wieder vor neue Herausforderungen zu stellen (etwa wechselnde Badges, Freischalten neuer Funktionen, Beschränkung der Zeitdauer für Ranglisten auf eine Woche/einen Monat) und ihnen neue Aufgaben zu erteilen, da ansonsten ein Gewöhnungseffekt einsetzt und das Spiel an Attraktivität verliert. Das bedeutet, dass Unternehmen den Spielverlauf beobachten und strategisch begleiten müssen. Das Konzept des persuasiven Designs, also der Überredung oder Überzeugung durch Botschaften oder die Darstellung der individuellen oder auch soziale Relevanz einer Aktivität, spielt hier eine wichtige Rolle (Werbach 2014). Eine Team-Challenge als beispielhaftes dramaturgisches Element kann genutzt werden, um das Engagement von Mitarbeitern zielorientiert zu steuern.

Das Netzwerk ist ein entscheidender Faktor in der Spielgestaltung bei Spielen im Multiplayer-Modus. Der Netzwerkcharakter motiviert etwa durch die Möglichkeit, Gruppen zu bilden und sozial eingebettet zu spielen. Auch Challenges können auf Basis von Netzwerken (Standort gegen Standort) initiiert werden. Der Netzwerkaspekt ist zudem die Grundlage für soziale Anreize (sozialer Vergleich etwa über Badge Sharing, Interaktion über Versand von Spieleinladungen).

\section{Ausblick}

Social Serious Games bieten Mitarbeitern die Möglichkeit, selbstbestimmt und am individuellen Bedarf orientiert zu lernen. Aus unmotivierenden und häufig als zeitraubend empfundenen Weiterbildungsmaßnahmen können auf diese Weise abwechslungsreiche und im Arbeitsalltag willkommene Aktivitäten werden. Die Unternehmensführung profitiert von einem gesteigerten Engagement und einer aktiven Beteiligung aller Mitarbeiter, da diese als Spieler selbst agieren müssen und die Rolle 
Abb. 2 Abhängigkeiten dynamische Rahmenbedingungen in Social Serious Games

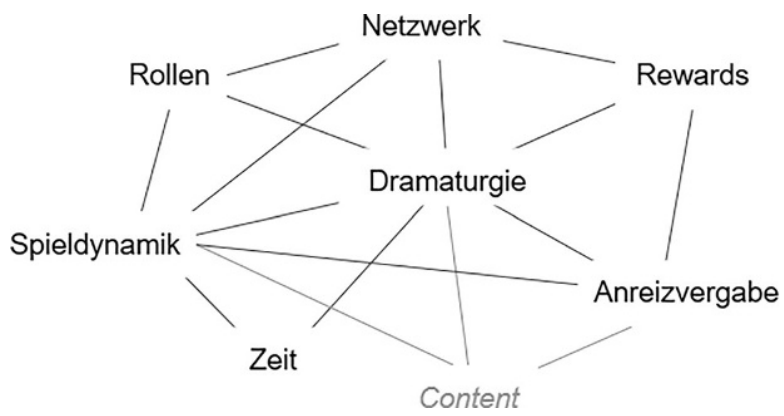

des bloßen Wissensrezipienten verlassen. Die soziale Einbettung des Lernprozesses durch anknüpfende Netzwerk-Funktionalitäten (Spieleinladungen, Teilen von Ergebnissen mit der Community) erzeugt Nachhaltigkeit durch gruppendynamische Prozesse.

Der vorliegende Beitrag hat deutlich gemacht, dass der Erfolg der Einführung neuer Weiterbildungskonzepte an Rahmenbedingungen geknüpft ist. Diese vielfältigen Aspekte sind oftmals miteinander verknüpft und können nicht isoliert sondern müssen stets in ihrer Gesamtheit betrachtet werden (Abb. 2).

Ein zusätzlich zu den zuvor beschriebenen Aspekten aufgeführter Erfolgsfaktor ist der Content. Die Art und Weise wie Inhalte generiert, identifiziert, aktualisiert oder auch entfernt werden hat gleichfalls einen immensen Einfluss auf das Spielerlebnis. Welche Möglichkeiten es etwa bei der Einbindung von Spielern in den Content Creation Prozess gibt, soll deshalb Gegenstand weiterführender Forschungsarbeiten sein.

Förderung Dieser Publikation liegen Arbeiten zugrunde, welche als Teil des Forschungs- und Entwicklungsprojekts SB:Digital im Rahmen des Programms „Zukunft der Arbeit“ [FKZ 02L15A070, Laufzeit: 01.04.2017 bis 31.03.2020] vom Bundesministerium für Bildung und Forschung (BMBF) und dem Europäischen Sozialfonds (ESF) gefördert und vom Projektträger Karlsruhe (PTKA) betreut wird.

GEFÖRDERT VOM
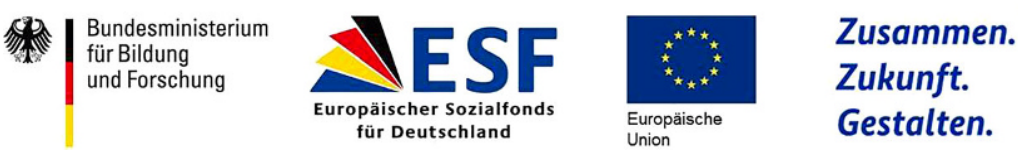

Open Access Dieser Artikel wird unter der Creative Commons Namensnennung 4.0 International Lizenz (http://creativecommons.org/licenses/by/4.0/deed.de) veröffentlicht, welche die Nutzung, Vervielfältigung, Bearbeitung, Verbreitung und Wiedergabe in jeglichem Medium und Format erlaubt, sofern Sie den/die ursprünglichen Autor(en) und die Quelle ordnungsgemäß nennen, einen Link zur Creative Commons Lizenz beifügen und angeben, ob Änderungen vorgenommen wurden.

\section{Literatur}

Ardichvili A, Page V, Wentling T (2003) Motivation and barriers to participation in virtual knowledgesharing communities of practice. J Knowl Manag 7(1):64-77. https://doi.org/10.1108/ 13673270310463626

Bartle R (1996) Hearts, clubs, diamonds, spades: players who suit MUDs. J Mud Res 1(1). http://www. mud.co.uk/richard/hcds.htm. Zugegriffen: 19.12.2018. 
Chou Y (2013) The six contextual types of rewards in gamification. http://yukaichou.com/marketinggamification/six-context-types-rewards-gamification/. Zugegriffen: 29. Nov. 2018

Csikszentmihalyi M (1990) Flow. The psychology of optimal experience. Harper \& Row, New York

Deterding S, Khaled R, Nacke L, Dixon D (2011) Gamification: toward a definition. CHI 2011 Gamification Workshop, Vancouver

McAfee A (2006) Enterprise 2.0. The dawn of emergent collaboration. Engineering Management Review, IEEE 34 (3): 38. https://doi.org/10.1109/EMR.2006.261380

Michl W (2015) Erlebnispädagogik, 3. Aufl. UTB Profile 3049. Reinhardt, München, Basel

Nacke L, Bateman C, Mandryk R (2011) BrainHex: preliminary results from a neurobiological gamer typology survey. In: Hutchison D, Kanade T, Kittler J et al (Hrsg) Entertainment computing ICEC 2011. Lecture notes in computer science, Bd. 6972. Springer, Berlin, Heidelberg, S 288-293

Peffers K, Tuunanen T, Rothenberger M, Chatterjee S (2007) A design science research methodology for information systems research. J Manag Inf Syst 24(3):45-77. https://doi.org/10.2753/MIS07421222240302

Scott K, Sorokti K, Merrell J (2016) Learning "beyond the classroom” within an enterprise social network system. Internet High Educ 29:75-90. https://doi.org/10.1016/j.iheduc.2015.12.005

Shpakova A, Dörfler V, Macbryde J (2016) The role(s) of gamification in knowledge management. EURAM 2016, S 1-40

Stieglitz S (2015) Gamification - Vorgehen und Anwendung. HMD 52(6):816-825. https://doi.org/10. 1365/s40702-015-0185-6

Ulich E (2013) Arbeitssysteme als soziotechnische Systeme - eine Erinnerung. J Psychol Alltagshandelns 6(1):4-12

Werbach K (2014) (Re)defining gamification: a process approach. In: Spagnolli A, Chittaro L, Gamberini L (Hrsg) Persuasive technology PERSUASIVE 2014. Lecture notes in computer science, Bd. 8462. Springer, Cham https://doi.org/10.1007/978-3-319-07127-5_23 